\title{
A Comparison of Stairs and Escalators in Virtual Reality
}

\author{
J. Hugh Wright \\ Rhodes College \\ Memphis, Tennessee \\ wrijh-20@rhodes.edu
}

\author{
Benjamin Yaffee \\ Rhodes College \\ Memphis, Tennessee \\ yafbl-21@rhodes.edu
}

\author{
Betsy Williams-Sanders \\ Rhodes College \\ Memphis, Tennessee \\ sandersb@rhodes.edu
}

\begin{abstract}
In this poster we present an in-progress study to compare the usage of simulated escalators with simulated stairs within a virtual reality (VR) environment. We found no existing research that examines the usage of escalators in VR. Past research into virtual stairs has examined how to better simulate stairs in a virtual environment (VE) by using external tools to allow an individual to more closely match real world movements. With virtual stairs, the user moves forward horizontally while the virtual avatar moves horizontally and vertically. With escalators, the user may stand in place to move the same distance within the virtual space, while also closely mimicking the movements they would make on an actual escalator without requiring additional tools. This experiment will test if the advantage of escalators requiring less real-world movement is offset by other factors, such as nausea, general discomfort, and the presence of the participant during the simulation.
\end{abstract}

\section{CCS CONCEPTS}

- Computing methodologies $\rightarrow$ Virtual reality; $\bullet$ Applied computing $\rightarrow$ Psychology.

\section{KEYWORDS}

virtual reality, virtual environments, locomotion, escalators, stairs

ACM Reference Format:

J. Hugh Wright, Benjamin Yaffee, and Betsy Williams-Sanders. 2019. A Comparison of Stairs and Escalators in Virtual Reality. In Symposium on Spatial User Interaction (SUI '19), October 19-20, 2019, New Orleans, LA, USA. ACM, New York, NY, USA, 2 pages. https://doi.org/10.1145/3357251.3358759

\section{BACKGROUND AND PURPOSE}

Visually induced motion sickness occurs when individuals who are stationary in reality perceive movement in a virtual environment [1]. This is the case when riding a virtual escalator. Furthermore, while riding an escalator an individual is not in control of her movements, and a lack of control is also associated with more severe simulator sickness [4]. Because of this one might expect that climbing virtual stairs, which gives an individual significantly more control over their speed and movements and is accompanied by real world movement, would cause significantly less simulator sickness than riding a virtual escalator, and give a much larger feeling of presence. However, riding an escalator is a real-world situation

Permission to make digital or hard copies of part or all of this work for personal or classroom use is granted without fee provided that copies are not made or distributed for profit or commercial advantage and that copies bear this notice and the full citation on the first page. Copyrights for third-party components of this work must be honored.

For all other uses, contact the owner/author(s).

SUI '19, October 19-20, 2019, New Orleans, LA, USA

(C) 2019 Copyright held by the owner/author(s)

ACM ISBN 978-1-4503-6975-6/19/10.

https://doi.org/10.1145/3357251.3358759
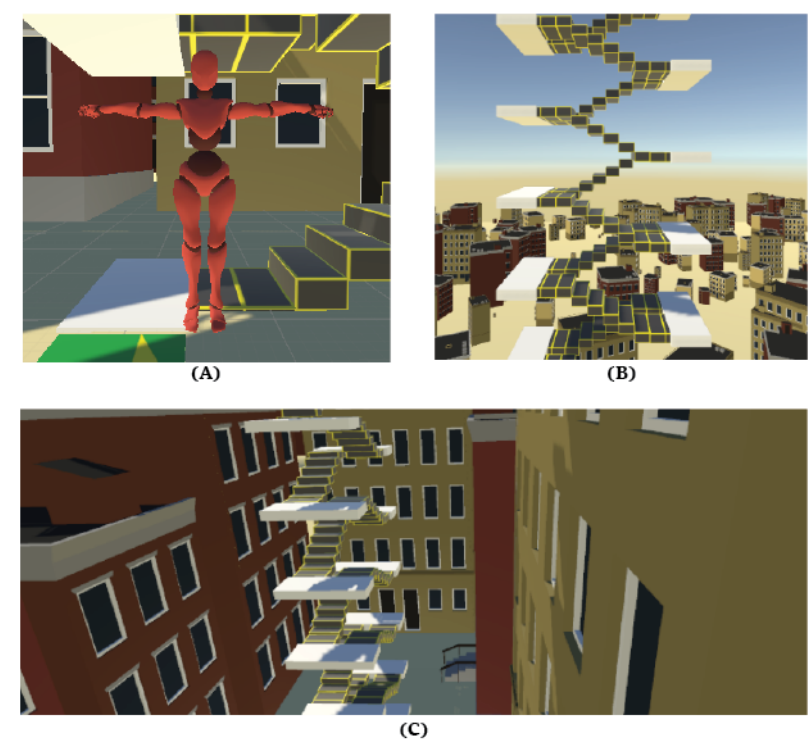

Figure 1: Screenshots taken of our virtual environment. The user's virtual avatar, (A). The tower of escalators used in the experiment, $(B)$ and $(C)$.

where an individual expects to be moved constantly after boarding. It is unclear whether the real-world association of riding an escalator counteracts the lack of real-world motion and control, and therefore whether the use of virtual escalators is a viable method of vertical locomotion in a VE. There are significant drawbacks to using virtual stairs for vertical locomotion. Since an individual generally walks forward when climbing virtual stairs, virtual stairs require additional space or treadmills which are not needed to simulate an escalator. Furthermore, on an escalator it is not unnatural for an individual to remain stationary until she reaches her destination, whereas with stairs it is difficult to match the real-world motions and tangible feeling of climbing when standing on a flat environment. To counteract this, past research has considered the use of external tools such as motorized stilts [3] or raised bumps on the floor [2] to provide tactile feedback and increase presence. A virtual escalator might provide an entirely digital alternative for when external tools are not available, or space is limited.

\section{MATERIALS}

For our experiment, we will use a Vive Pro Head Mounted Display (HMD) with a wireless connection along with Vive foot trackers and hand-held controllers. The HMD has a resolution of $1440 \mathrm{x}$ 1600 for each eye. The virtual environment (VE) that will be used 
in our experiment was created in Unity. Our VE contains a tower, shown in Figure 1, made up of zigzagging escalators. The tower is surrounded by low poly buildings which were downloaded from the Unity asset store and have an average height of 12 meters. The tower is 220 meters tall and made up of 200 zig-zagging flights of escalators (which can be frozen in place to create stairs). Each escalator ascends at $30^{\circ}$ and is 3.8 meters long, including flat sections for boarding and exiting the escalator. Participants in the experiment will be embodied by the red robot shown in Figure 1A. Real world movements are mapped to the virtual avatar using the Simple IK (Inverse Kinematics) plugin, downloaded from the Unity asset store.

\section{EXPERIMENTAL DESIGN}

This experiment will use a within-subject experimental design to test four methods of vertical locomotion: Riding an escalator moving at $0.45 \mathrm{~m} / \mathrm{s}$ (the speed of an escalator in the New York subway), riding an escalator moving at $0.80 \mathrm{~m} / \mathrm{s}$ (the speed of an escalator in Hong Kong), climbing on stairs, and climbing on an escalator moving at $0.45 \mathrm{~m} / \mathrm{s}$. While riding an escalator the subjects will be asked to not move forward on the escalator; while climbing they will be given the option to ascend faster by moving forward in reality. When climbing the stairs or escalators the user's viewpoint is raised smoothly based on the position of the head tracker. We intend to use a sample size of 12 volunteers taken from our college campus. We will have them use each method of vertical locomotion in a random order. For each condition we will have them move up for two minutes and down for two minutes. Prior to testing a new condition each subject will be given time to practice moving by ascending and descending three flights of escalators or stairs. After moving up or down for two minutes they will guess the height of the structure they were moving on (to test their perception of height for the different conditions). This test will be performed by allowing the subject to change the height of a virtual escalator in front of them. This virtual escalator will initially be flat and the subject will be able to raise or lower the height of the structure by raising or lowering their Vive controller. After moving up and down for each condition, the participants will fill out a questionnaire to test presence and simulator sickness.

\section{PILOT STUDY}

We recruited three volunteers to participate in a pilot study. All three were males between 18 and 22 and had extensive experience using VR. Our pilot study indicated that presence, particularly a feeling of moving up or down, was higher for escalator-based conditions if the participant remained stationary while riding. It is unclear whether presence was significantly higher when the participant was not stationary on the escalator. Simulator sickness levels and the perceived height of the structures did not noticeably change across all conditions.

\section{ACKNOWLEDGMENTS}

This material is based upon work supported by the National Science Foundation under grant 1351212. Any opinions, findings, and conclusions or recommendations expressed in this material are those of the authors and do not necessarily reflect the views of the sponsors.

\section{REFERENCES}

[1] Lawrence J. Hettinger and Gary E. Riccio. 1992. Visually Induced Motion Sickness in Virtual Environments. Presence: Teleoperators and Virtual Environments 1, 3 (1992), 306-310. https://doi.org/10.1162/pres.1992.1.3.306

[2] Ryohei Nagao, Keigo Matsumoto, Takuji Narumi, Tomohiro Tanikawa, and Michitaka Hirose. 2017. Infinite stairs: simulating stairs in virtual reality based on visuo-haptic interaction. In ACM SIGGRAPH 2017 Emerging Technologies. ACM, ACM, 14.

[3] Dominik Schmidt, Rob Kovacs, Vikram Mehta, Udayan Umapathi, Sven Köhler, Lung-Pan Cheng, and Patrick Baudisch. 2015. Level-Ups: Motorized Stilts That Simulate Stair Steps in Virtual Reality. In Proceedings of the 33rd Annual ACM Conference on Human Factors in Computing Systems (CHI '15). ACM, New York, NY, USA, 2157-2160. https://doi.org/10.1145/2702123.2702253

[4] Kay M. Stanney and Phillip Hash. 1998. Locus of User-Initiated Control in Virtual Environments: Influences on Cybersickness. Presence: Teleoperators and Virtual Environments 7, 5 (1998), 447-459. https://doi.org/10.1162/105474698565848 